\title{
Discrete information from CHL black holes
}

\author{
Ashoke Sen \\ Harish-Chandra Research Institute, \\ Chhatnag Road, Jhusi, Allahabad 211019, India \\ LPTHE, Universite Pierre et Marie Curie Paris 6, \\ 4 Place Jussieu, 75252 Paris Cedex 05, France \\ E-mail: sen@mri.ernet.in
}

Abstract: $A d S_{2} / C F T_{1}$ correspondence predicts that the logarithm of a $\mathbb{Z}_{N}$ twisted index over states carrying a fixed set of charges grows as $1 / N$ times the entropy of the black hole carrying the same set of charges. In this paper we verify this explicitly by calculating the microscopic $\mathbb{Z}_{N}$ twisted index for a class of states in the CHL models. This demonstrates that black holes carry more information about the microstates than just the total degeneracy.

Keywords: Black Holes in String Theory, D-branes

ARXIV EPRINT: 1002.3857 


\section{Contents}

1 Introduction and summary 1

2 The counting 5

$\begin{array}{lll}3 & \text { Asymptotic growth } & 9\end{array}$

$\begin{array}{lll}4 & \text { Conclusion } & 10\end{array}$

$\begin{array}{ll}\text { A Explicit computation of } c_{b}^{\left(r, s ; r^{\prime}, s^{\prime}\right)} & 11\end{array}$

B Threshold integral representation of $\widetilde{\Phi} \quad 12$

$\begin{array}{ll}\text { C Zeroes of } \widetilde{\Phi} & 15\end{array}$

\section{Introduction and summary}

CHL models $[1,2]$ in four dimensions with $\mathcal{N}=4$ supersymmetry have proved to be a rich arena for studying the physics of black holes [3-9]. On the one hand they have as much supersymmetry and hence as much control as the toroidally compactified heterotic string theory. On the other hand they have different effective actions beyond the supergravity approximation and hence make different predictions for the entropy of BPS black holes beyond the leading order result of $[10,11]$. Thus they provide us with more data points at which we can compare the macroscopic and microscopic predictions for the black hole entropy, pushing the observation of $[12,13]$ to greater accuracy. This comparison has been remarkably successful at the level of four derivative corrections to the effective action, reproducing complicated non-trivial functional dependence of the entropy on the charges on both sides. ${ }^{1}$ Indeed, most of the results on black holes in heterotic string theory on $T^{6}[6,18-26]$ have now been generalized to the case of CHL models.

In this paper we shall make use of the CHL model to explore another aspect of black holes. Based on $A d S_{2} / C F T_{1}$ correspondence [27, 28] it was argued in [29] that if a theory has a $\mathbb{Z}_{N}$ symmetry that cannot be regarded as part of a $\mathrm{U}(1)$ gauge transformation, and if we pick a black hole carrying $\mathrm{U}(1)$ charges which are invariant under this $\mathbb{Z}_{N}$

\footnotetext{
${ }^{1}$ We should add a note of caution that this comparison requires us to make assumption of certain nonrenormalization results which have not been proven. In particular it assumes that at the level of four derivative terms the Gauss-Bonnet terms (or their supersymmetric completion given in [14-17]) in the action are sufficient to calculate the correction to the black hole entropy. The analysis in this paper does not require us to make any such assumption.
} 
transformation, ${ }^{2}$ then the logarithm of the trace of the $\mathbb{Z}_{N}$ generator over the microstates of the black hole grows as $1 / N$ times the entropy of the black hole. ${ }^{3}$ This can be made more concrete for BPS black holes in supersymmetric string theories by working with protected helicity trace index. In the context of $\mathcal{N}=4$ supersymmetric string theories in four dimensions the relevant twisted index is the 6th helicity trace index $[29,32,33]$ :

$$
B_{6}^{g}(\vec{q})=\frac{1}{6 !} \operatorname{Tr}_{\vec{q}}\left\{(-1)^{2 h}(2 h)^{6} g\right\}
$$

where the trace is taken over all states carrying a fixed set of charges $\vec{q}, h$ is the third component of the angular momentum of the state in its rest frame, and $g$ is the generator of a $\mathbb{Z}_{N}$ symmetry which leaves $\vec{q}$ invariant. This index receives contribution from $1 / 4$ BPS states in this theory which describe large black holes with near horizon $A d S_{2} \times S^{2}$ geometry. In this case the analysis of [29] applies and tells us that

$$
\left|B_{6}^{g}(\vec{q})\right| \sim \exp \left[S_{B H}(\vec{q}) / N\right],
$$

where $S_{B H}(\vec{q})$ is the entropy of an extremal black hole carrying charge $\vec{q}$. We shall not review the arguments of [29] here; but the central idea is that in computing the contribution to (1.2) from the horizon of the black hole the leading saddle point corresponding to the $A d S_{2} \times S^{2}$ near horizon geometry does not contribute. However a $\mathbb{Z}_{N}$ orbifold of $A d S_{2} \times S^{2}[28,34,35]$, whose asymptotic geometry coincides with that of the original near horizon geometry of the black hole, contributes and gives the answer $\exp \left[S_{B H} / N\right]$ in the semiclassical limit.

While (1.2) follows almost trivially from the $A d S_{2} / C F T_{1}$ correspondence, it is quite striking from the point of view of the microscopic theory. For large black holes the right hand side of (1.2) is much smaller than the untwisted helicity trace index carrying the same charges, since the latter is given by $\exp \left[S_{B H}(\vec{q})\right]$. What this tells us is that in a given charge sector the microstates of different $g$ eigenvalues come in almost equal numbers so that the sum weighted by $g$ is much smaller than the total number of states. This was explicitly verified in [29] by deriving the microscopic formula for this twisted index in toroidally compactified heterotic and type II string theories and then studying their asymptotic behaviour. ${ }^{4}$

Given the unusual nature of this macroscopic prediction, it is important to test this in as many examples as possible. In this paper we shall verify this in the context of CHL

\footnotetext{
${ }^{2}$ Consider for example heterotic string theory compactified on $T^{6}$. In this theory we have a $\mathbb{Z}_{2}$ symmetry that exchanges the two $E_{8}$ factors. If we use this $\mathbb{Z}_{2}$ transformation to construct a twisted index we must restrict to states which carry equal charges under the two $E_{8}$ factors (more precisely their Cartan subalgebras).

${ }^{3}$ For a $\mathbb{Z}_{N}$ group that can be regarded as a subgroup of a spontaneously broken U(1) gauge group, the possibility of hair modes containing information about the $\mathbb{Z}_{N}$ quantum numbers was explored in [30, 31]. In contrast the $\mathbb{Z}_{N}$ groups we discuss here cannot be regarded as a subgroup of a spontaneously broken $\mathrm{U}(1)$ symmetry. Also our goal here is quite different from the one of [30, 31].

${ }^{4}$ Even though type II string theory on $T^{6}$ has $\mathcal{N}=8$ supersymmetry, only an $\mathcal{N}=4$ subgroup of this commutes with $g$. Thus effectively we can analyze it in the same way as in an $\mathcal{N}=4$ supersymmetric theory.
} 
models. The construction of the CHL models that we shall analyze proceeds as follows. We begin with type IIB string theory on $\mathcal{M} \times S^{1} \times \widetilde{S}^{1}$ where $\mathcal{M}$ is either $\mathrm{K} 3$ or $T^{4}$ and go to a special subspace of the moduli space of $\mathcal{M}$ where the theory has a geometric $\mathbb{Z}_{M} \times \mathbb{Z}_{N}$ symmetry that commutes with 16 supersymmetry generators of the theory. An extensive list of possible symmetries of this type can be found in $[36,37]$. Note also that a $Z_{M N}$ group with $M$ and $N$ relatively prime can be considered as a $Z_{M} \times Z_{N}$ group for the purpose of our analysis. Let us denote by $g_{M}$ and $g_{N}$ the generators of $\mathbb{Z}_{M}$ and $\mathbb{Z}_{N}$ respectively. We now take an orbifold of this theory by a symmetry that involves $1 / M$ unit of translation along the circle $S^{1}$ accompanied by the transformation $g_{M}$. This gives a theory with $\mathcal{N}=4$ supersymmetry in four dimensions and the $\mathbb{Z}_{N}$ group generated by $g_{N}$ is a symmetry of this theory. We now consider a $g_{N}$ invariant charge vector $\vec{q}$ in this theory and define the index

$$
d(\vec{q})=-\frac{1}{6 !} \operatorname{Tr}_{\vec{q}}\left(e^{2 \pi i h}(2 h)^{6} g_{N}\right)
$$

where the trace is taken over all states carrying the charge $\vec{q}$. Eq. (1.2) now translates to

$$
|d(\vec{q})| \sim \exp \left[S_{B H}(\vec{q}) / N\right],
$$

for large charges. Our goal will be to verify this by explicit computation of $d(\vec{q})$ in the microscopic theory.

Since the explicit counting of states involves technical details, we shall take this opportunity to summarize the results of our analysis. We use a convention in which the coordinate radius of the original circle $S^{1}$ before orbifolding is $2 \pi M$ so that the orbifold action involves translation by $2 \pi$ along $S^{1}$ accompanied by $g_{M}$. In this convention the minimum amount of momentum along $S^{1}$ is $1 / M$. We focus on states carrying one unit of KK monopole charge associated with the circle $\widetilde{S}^{1}$, one unit of D5-brane charge wrapped on $\mathcal{M} \times S^{1}, Q_{1}$ units of D1-brane charge wrapped on $S^{1}$, left-moving momentum $n / M$ along $S^{1}$ and $J$ units of momentum along $\widetilde{S}^{1}$, and define

$$
Q^{2}=2 n / M, \quad P^{2}=2 Q_{1}, \quad Q \cdot P=J .
$$

In this case our result of $d(\vec{q})$ is given by

$$
d(\vec{q})=\frac{1}{M}(-1)^{Q . P+1} \int_{\mathcal{C}} d \rho d \sigma d v e^{-\pi i\left(M \rho Q^{2}+\sigma P^{2} / M+2 v Q . P\right)} \frac{1}{\widetilde{\Phi}(\rho, \sigma, v)} .
$$

Here $\mathcal{C}$ is a three real dimensional subspace of the three complex dimensional space labelled by $\left(\rho=\rho_{1}+i \rho_{2}, \sigma=\sigma_{1}+i \sigma_{2}, v=v_{1}+i v_{2}\right)$ given by

$$
\begin{aligned}
\rho_{2} & =M_{1}, \quad \sigma_{2}=M_{2}, \quad v_{2}=M_{3}, \\
0 & \leq \rho_{1} \leq 1, \quad 0 \leq \sigma_{1} \leq M,
\end{aligned}
$$

$M_{1}, M_{2}, M_{3}$ being large but fixed positive numbers satisfying

$$
M_{1} M_{2}>M_{3}^{2} .
$$


The function $\widetilde{\Phi}(\rho, \sigma, v)$ is a modular form of a subgroup of $\operatorname{Sp}(2, \mathbb{Z})$, given by

$$
\begin{aligned}
\widetilde{\Phi}(\rho, \sigma, v) & =e^{2 \pi i(\widetilde{\alpha} \rho+\widetilde{\gamma} \sigma+\widetilde{\beta} v)} \prod_{b=0}^{1} \prod_{r=0}^{N-1} \prod_{r^{\prime}=0}^{M-1} \prod_{\substack{k \in \mathbb{Z}+\frac{r^{\prime}}{M}, l \in \mathbb{Z}, j \in 2 \mathbb{Z}+b \\
k, l \geq 0, j<0 \\
\text { for } k=l=0}}\left[1-e^{2 \pi i r / N} e^{2 \pi i(k \sigma+l \rho+j v)}\right]^{a} \\
a & \equiv \sum_{s=0}^{N-1} \sum_{s^{\prime}=0}^{M-1} e^{-2 \pi i\left(s^{\prime} l / M+r s / N\right)} c_{b}^{\left(0, s ; r^{\prime}, s^{\prime}\right)}\left(4 k l-j^{2}\right)
\end{aligned}
$$

where the coefficients $c_{b}^{\left(r, s ; r^{\prime}, s^{\prime}\right)}$ are defined via the equation:

$$
\begin{aligned}
& \sum_{b=0}^{1} \sum_{j \in 2 \mathbb{Z}+b, n \in \mathbb{Z} / M N} c_{b}^{\left(r, s ; r^{\prime}, s^{\prime}\right)}\left(4 n-j^{2}\right) e^{2 \pi i(n \tau+j z)} \\
& \quad=\frac{1}{M N} \operatorname{Tr}_{R R ; g_{M}^{r^{\prime}} g_{N}^{r}}\left(g_{M}^{s^{\prime}} g_{N}^{s}(-1)^{J_{L}+J_{R}} e^{2 \pi i\left(\tau L_{0}-\bar{\tau} \bar{L}_{0}\right)} e^{2 \pi i J_{L} z}\right)
\end{aligned}
$$

The trace is taken over all the $g_{M}^{r^{\prime}} g_{N}^{r}$ twisted RR sector states in the $(4,4)$ superconformal $\mathrm{CFT}_{2}$ with target space $\mathcal{M} . L_{0}$ and $\bar{L}_{0}$ are the left and right-moving Virasoro generators and $J_{L} / 2$ and $J_{R} / 2$ are the generators of the $\mathrm{U}(1)_{L} \times \mathrm{U}(1)_{R}$ subgroup of the $\mathrm{SU}(2)_{L} \times \mathrm{SU}(2)_{R}$ R-symmetry group of this $\mathrm{CFT}_{2}$. An algorithm for explicitly computing the right hand side of (1.10) has been outlined in appendix A. The coefficients $\widetilde{\alpha}, \widetilde{\beta}, \widetilde{\gamma}$ are given by

$$
\begin{aligned}
& \widetilde{\alpha}=\frac{1}{24 M} Q_{0,0}-\frac{1}{2 M} \sum_{s^{\prime}=1}^{M-1} Q_{0, s^{\prime}} \frac{e^{-2 \pi i s^{\prime} / M}}{\left(1-e^{-2 \pi i s^{\prime} / M}\right)^{2}}, \\
& \widetilde{\beta}=1 \\
& \widetilde{\gamma}=\frac{1}{24 M} \chi(\mathcal{M})=\frac{1}{24 M} Q_{0,0},
\end{aligned}
$$

where

$$
Q_{r^{\prime}, s^{\prime}}=M N\left(c_{0}^{\left(0,0 ; r^{\prime}, s^{\prime}\right)}(0)+2 c_{1}^{\left(0,0 ; r^{\prime}, s^{\prime}\right)}(-1)\right) .
$$

Eqs. (1.10), (1.11) define all the quantities which appear in the definition of $\widetilde{\Phi}$. The only ambiguity that remains in computing the right hand side of (1.6) is the choice of the integration contour encoded in the choice of $\left(M_{1}, M_{2}, M_{3}\right)$. As is well known by now, this ambiguity is related to the phenomenon of wall crossing [38-41]. Different choices of $M_{1}$, $M_{2}$ and $M_{3}$ give the value of $d(\vec{q})$ for different values of the asymptotic moduli. However the ambiguity in the value of $d(\vec{q})$ that it introduces is sufficiently small so as not to affect our analysis, and hence we shall ignore it in our subsequent discussion.

Given the result (1.6) for $d(\vec{q})$ we can find its behaviour for large $Q^{2}, P^{2}$ and Q.P by standard method $[3,6,18,19]$. The result is that $d(\vec{q})$ behaves as

$$
d(\vec{q}) \sim \exp \left[\pi \sqrt{Q^{2} P^{2}-(Q . P)^{2}} / N\right] .
$$

Since in this limit a black hole of charge $\vec{q}$ has entropy $[10,11]$

$$
S_{B H}(\vec{q}) \simeq \pi \sqrt{Q^{2} P^{2}-(Q . P)^{2}},
$$


we see that the microscopic result (1.13) is in perfect agreement with the macroscopic prediction (1.4).

Finally we would like to remark that even though we have presented our analysis for the index $\operatorname{Tr}\left((-1)^{2 h}(2 h)^{6} g_{N}\right)$, we can repeat the analysis with $g_{N}$ replaced by $\left(g_{N}\right)^{b}$ for any integer $b$. In this case the role of $N$ is played by the order of $\left(g_{N}\right)^{b}$, and in all the formulæ we simply have to replace $g_{N}$ by $\left(g_{N}\right)^{b}$. This in turn allows us to compute the index $\operatorname{Tr}\left((-1)^{2 h}(2 h)^{6}\right)$ for states carrying a definite $g_{N}$ eigenvalue $e^{2 \pi i a / N}$ using the combination

$$
\frac{1}{N} \sum_{b=0}^{N-1} e^{-2 \pi i a b / N} \operatorname{Tr}\left((-1)^{2 h}(2 h)^{6}\left(g_{N}\right)^{b}\right) .
$$

Thus our result can also be interpreted as the agreement between the macroscopic and the microscopic results for the helicity trace index over states carrying a definite $g_{N}$ charge.

\section{The counting}

The counting of states of the D1-D5-KK monopole system proceeds as in [6-8, 42]. We take the circle $S^{1}$ to be large compared to the size of $\mathcal{M}$ and regard the world-volume theory as a $1+1$ dimensional field theory living on $S^{1}$. Denoting by $d\left(Q_{1}, n, J\right)$ the twisted index (1.3) of states carrying charge labeled by $\left(Q_{1}, n, J\right)$ in the convention of section 1 , we define

$$
Z(\rho, \sigma, v)=\sum_{Q_{1}, n, J} e^{2 \pi i\left(Q_{1} \sigma / M+n \rho+v J\right)}(-1)^{J} d\left(Q_{1}, n, J\right)
$$

Proving (1.6) is now equivalent to proving that $Z=-1 / \widetilde{\Phi}$.

Since the are computing a protected index, it is independent of the moduli except for jumps across walls of marginal stability controlled by the wall crossing formula. Thus we can compute the index at a convenient corner of the moduli space. As in [6] we shall work in weakly coupled type IIB string theory in which case the twisted partition function $Z$ is given by the product of three twisted partition functions, - that of the excitations living on the KK monopole, that of the dynamics describing the overall motion of the D1-D5 system in the background of the KK monopole and that of the motion of the D1brane along the D5-brane. For each system we must keep the right-movers in their ground state and excite the left-movers in order to preserve supersymmetry. ${ }^{5}$ Since the fermion zero modes associated with the broken supersymmetries are automatically removed while computing the helicity trace (which is $B_{6}$ in this case since the system breaks 12 of the 16 supersymmetries), we shall ignore their contribution during the rest of our analysis.

We begin by analyzing the partition function of the KK monopole. The massless bosonic modes on the world-volume of the KK monopole arise from the motion along the three transverse directions and the components of the $p$-form fields along the product of the harmonic $(p-2)$-forms of $\mathcal{M}$ and the harmonic 2-form of the Taub-NUT space. The massless fermions are the goldstinos associated with the supersymmetries broken by the

\footnotetext{
${ }^{5}$ Here and elsewhere left-moving modes will refer to modes carrying momentum along the negative $S^{1}$ direction. Thus left-moving momentum $n$ will indicate momentum $-n$ along $S^{1}$.
} 
Kaluza-Klein monopole. In general one can show that the left-moving bosons and fermions are in one to one correspondence with the even and odd degree harmonic forms on $\mathcal{M}$ [42]. Furthermore their $\left(g_{M}, g_{N}\right)$ quantum numbers are also given by the $\left(g_{M}, g_{N}\right)$ eigenvalues of the harmonic forms on $\mathcal{M}$. Since the harmonic $(p, q)$ forms on $\mathcal{M}$ are in one to one correspondence with the $\mathrm{RR}$ sector ground states in the supersymmetric $\sigma$-model with target space $\mathcal{M}$ carrying $L_{0}=\bar{L}_{0}=0, J_{L}=(p-1), J_{R}=(q-1)$, it follows from (1.10) that the number of left-moving bosons minus the number of left-moving fermions on the KK monopole world-volume, carrying $g_{N}$ quantum number $e^{2 \pi i r / N}$ and $g_{M}$ quantum number $e^{2 \pi i k^{\prime} / M}$, is given by $[8,42]$

$$
\begin{gathered}
\frac{1}{M N} \sum_{s=0}^{N-1} \sum_{s^{\prime}=0}^{M-1} e^{-2 \pi i r s / N} e^{-2 \pi i k^{\prime} s^{\prime} / M} \operatorname{Tr}_{R R ; I}\left[(-1)^{J_{L}+J_{R}} g_{M}^{s^{\prime}} g_{N}^{s} \delta_{L_{0}, 0} \delta_{\bar{L}_{0}, 0}\right] \\
=\sum_{s=0}^{N-1} \sum_{s^{\prime}=0}^{M-1} e^{-2 \pi i r s / N} e^{-2 \pi i k^{\prime} s^{\prime} / M}\left(c_{0}^{\left(0, s ; 0, s^{\prime}\right)}(0)+2 c_{1}^{\left(0, s ; 0, s^{\prime}\right)}(-1)\right) .
\end{gathered}
$$

In arriving at $(2.2)$ we have used the fact that $c_{b}^{\left(r, s ; r^{\prime}, s^{\prime}\right)}(u)=0$ for $u<-1$. Now consider a mode carrying $g_{M}$ eigenvalue $e^{2 \pi i k^{\prime} / M}$ and left-moving momentum $l / M$ along $S^{1}$. The requirement of invariance under the simultaneous action of $g_{M}$ and $2 \pi$ translation along $S^{1}$ gives us the requirement that $l=k^{\prime} \bmod M$. Furthermore the contribution to the twisted index from these states will be weighted by the $g_{N}$ eigenvalue $e^{2 \pi i r / N}$. Thus the net contribution to the partition function from these modes is given by

$Z_{K K}=e^{-2 \pi i \widetilde{\alpha} \rho} \prod_{r=0}^{N-1} \prod_{l=1}^{\infty}\left(1-e^{2 \pi i r / N} e^{2 \pi i l \rho}\right)^{-\sum_{s=0}^{N-1} \sum_{s^{\prime}=0}^{M-1} e^{-2 \pi i r s / N} e^{-2 \pi i l s^{\prime} / M}\left(c_{0}^{\left(0, s ; 0, s^{\prime}\right)}(0)+2 c_{1}^{\left(0, s ; 0, s^{\prime}\right)}(-1)\right)}$.

Here the term $e^{-2 \pi i \widetilde{\alpha} \rho}$ reflects the effect of the momentum carried by the ground state of the Kaluza-Klein monopole. The analysis of [8, 42] gives

$$
\begin{aligned}
\widetilde{\alpha} & =\frac{1}{24 M} Q_{0,0}-\frac{1}{2 M} \sum_{s^{\prime}=1}^{M-1} Q_{0, s^{\prime}} \frac{e^{-2 \pi i s^{\prime} / M}}{\left(1-e^{-2 \pi i s^{\prime} / M}\right)^{2}}, \\
Q_{r^{\prime}, s^{\prime}} & \equiv M N\left(c_{0}^{\left(0,0 ; r^{\prime}, s^{\prime}\right)}(0)+2 c_{1}^{\left(0,0 ; r^{\prime}, s^{\prime}\right)}(-1)\right) .
\end{aligned}
$$

Next we turn to the dynamics of the overall motion of the D1-D5 system in the KK monopole background. The dynamics in the transverse direction is independent of whether we are working with $K 3$ or $T^{4}$. Furthermore these modes do not carry any $g_{N}$ or $g_{M}$ quantum numbers; thus the contribution from these modes to the partition function is universal. The result is $[8,42]$

$$
\begin{aligned}
& -e^{-2 \pi i v}\left(1-e^{-2 \pi i v}\right)^{-2} \\
& \quad \prod_{\substack{l \in M \mathbb{Z} \\
l>0}}\left\{\left(1-e^{2 \pi i l \rho}\right)^{4}\left(1-e^{2 \pi i l \rho+2 \pi i v}\right)^{-2}\left(1-e^{2 \pi i l \rho-2 \pi i v}\right)^{-2}\right\} .
\end{aligned}
$$

The first line represents the contribution from the zero mode dynamics that binds the D1D5 system to the KK monopole [6, 43, 44], and the second line represents the contribution 
from the oscillators. The last two terms in the second line of (2.5) represent the contribution from the four left-moving bosonic modes representing transverse oscillation of the D1-D5 system whereas the first factor in the same line represents contribution from the left-moving fermionic modes. ${ }^{6}$ In arriving at (2.5) one needs to use the fact that in the presence of the KK monopole background, the momentum along $\widetilde{S}^{1}$ appears as the angular momentum $2 J_{L}$ for the D1-D5 system where $J_{L}$ is the generator of the $\mathrm{U}(1)_{L} \subset \mathrm{SU}(2)_{L}$ subgroup of the rotation group in transverse space [45]. The $v$ dependence of (2.5) then follows from the fact that the bosonic modes, transforming as a vector of the transverse rotation group $\mathrm{SU}(2)_{L} \times \mathrm{SU}(2)_{R}$, carry $J_{L}= \pm 1$ while the fermionic modes are neutral under $\mathrm{U}(1)_{L}$ as a consequence of footnote 6 .

For $\mathcal{M}=T^{4}$ we also have four additional bosonic modes arising from the Wilson lines on the D5-brane along $T^{4}$ and four additional fermionic modes. In order to find the contribution to the partition function from these modes we need to know the action of $g_{M}$ and $g_{N}$ on these modes. If $z_{1}$ and $z_{2}$ denote the complex coordinates on $T^{4}$ then in order to preserve supersymmetry both $g_{M}$ and $g_{N}$ must act as equal and opposite rotation of $z_{1}$ and $z_{2}$, possibly accompanied by shifts. We shall assume for definiteness that $g_{M}$ and $g_{N}$ induce respectively $2 \pi / M$ and $2 \pi / N$ rotations on these coordinates:

$$
\begin{gathered}
g_{M}:\left(d z_{1}, d z_{2}\right) \rightarrow\left(e^{2 \pi i / M} d z_{1}, e^{-2 \pi i / M} d z_{2}\right), \\
g_{N}:\left(d z_{1}, d z_{2}\right) \rightarrow\left(e^{2 \pi i / N} d z_{1}, e^{-2 \pi i / N} d z_{2}\right) .
\end{gathered}
$$

(2.6) represents the action of $g_{M}$ and $g_{N}$ on the Wilson line variables. Furthermore the Wilson lines are neutral under the rotation group in the transverse space and hence carry $J_{L}=0$. The result of footnote 6 now tells us that the additional fermionic modes on the D1D5 system, which arise for $\mathcal{M}=T^{4}$, transform in the same way under $g_{M}$ and $g_{N}$, and carry $J_{L}= \pm 1$ uncorrelated with their $\left(g_{M}, g_{N}\right)$ quantum numbers [8, 42]. Thus the contribution from these additional modes to the twisted partition function is given by

$$
\begin{aligned}
& \prod_{\substack{l \in M \mathbb{Z}_{+} \\
l>0}}\left(1-e^{2 \pi i / N} e^{2 \pi i l \rho}\right)^{-2} \prod_{\substack{l \in M \mathbb{Z} \\
l>0}}\left(1-e^{-2 \pi i / N} e^{2 \pi i l \rho}\right)^{-2} \prod_{\substack{l \in M \mathbb{Z}+1 \\
l>0}}\left(1-e^{2 \pi i / N} e^{2 \pi i l \rho+2 \pi i v}\right) \\
& \prod_{\substack{l \in M \mathbb{Z}_{+} \\
l>0}}\left(1-e^{2 \pi i / N} e^{2 \pi i l \rho-2 \pi i v}\right) \prod_{\substack{l \in M \mathbb{Z}_{-} \\
l>0}}\left(1-e^{-2 \pi i / N} e^{2 \pi i l \rho+2 \pi i v}\right) \prod_{\substack{l \in M \mathbb{Z}-1 \\
l>0}}\left(1-e^{-2 \pi i / N} e^{2 \pi i l \rho-2 \pi i v}\right) .
\end{aligned}
$$

The first two factors come from the bosonic modes and the last four factors arise from the fermionic modes whose contribution have not already been included in (2.5). The only new ingredient in this formula compared to that in $[8,42]$ is the insertion of the factors of $e^{ \pm 2 \pi i / N},-$ these arise from the insertion of $g_{N}$ into the trace.

\footnotetext{
${ }^{6}$ These left-moving bosonic and fermionic modes, as well as those which contribute to (2.7), are paired by the unbroken superysymmetry transformations on the D1-D5 world volume in flat space-time which commute with $\mathbb{Z}_{M} \times \mathbb{Z}_{N}$, are charged under the $\mathrm{SU}(2)_{L}$ subgroup of the transverse rotation group, and act on the left-movers. Eventually when we place this system in the background of KK monopole this supersymmetry is broken since in the full system there is no supersymmetry acting on the left-movers. However this is still useful for determining the quantum numbers of the fermions from the known quantum numbers of the bosonic modes [8, 42].
} 
The product of (2.5) and (2.7) can be written in a compact form using the coefficients $c_{1}^{\left(0, s ; 0, s^{\prime}\right)}(-1)$. It follows from its definition, and the identification of the RR sector ground states in the SCFT with target space $\mathcal{M}$ carrying $\left(J_{L}, J_{R}\right)=(p-1, q-1)$ with the harmonic $(p, q)$ forms on $\mathcal{M}$, that $M N c_{1}^{\left(0, s ; 0, s^{\prime}\right)}(-1)$ represents trace over the $(0, q)$ forms on $\mathcal{M}$ weighted by $(-1)^{q} g_{N}^{s} g_{M}^{s^{\prime}}[8,42]$. On $K 3$ the only $(0, q)$ forms are $(0,0)$ forms and $(0,2)$ forms both of which are neutral under $g_{N}$ and $g_{M}$, while on $T^{4}$ we also have a pair of $(0,1)$ forms $d z_{1}$ and $d z_{2}$ which we have chosen to carry $\left(g_{N}, g_{M}\right)$ eigenvalues $\left(e^{ \pm 2 \pi i / N}, e^{ \pm 2 \pi i / M}\right)$. This gives

$$
\begin{aligned}
c_{1}^{\left(0, s ; 0, s^{\prime}\right)}(-1) & =\frac{2}{M N} \quad \text { for } \mathcal{M}=K 3 \\
& =\frac{1}{M N}\left(2-e^{2 \pi i s / N} e^{2 \pi i s^{\prime} / M}-e^{-2 \pi i s / N} e^{-2 \pi i s^{\prime} / M}\right) \quad \text { for } \mathcal{M}=T^{4}
\end{aligned}
$$

Using this we can express the total contribution to the partition function from the overall motion of the D1-D5 system in the Taub-NUT space, given by (2.5) for $\mathcal{M}=K 3$ and the product of (2.5) and (2.7) for $\mathcal{M}=T^{4}$, as

$$
\begin{aligned}
Z_{C M}= & -e^{-2 \pi i v} \prod_{l=1}^{\infty} \prod_{r=0}^{N-1}\left(1-e^{2 \pi i r / N} e^{2 \pi i l \rho}\right)^{2 \sum_{s=0}^{N-1} \sum_{s^{\prime}=0}^{M-1} e^{-2 \pi i l s^{\prime} / M} e^{-2 \pi i r s / N} c_{1}^{\left(0, s ; 0, s^{\prime}\right)}(-1)} \\
& \prod_{l=1}^{\infty} \prod_{r=0}^{N-1}\left(1-e^{2 \pi i r / N} e^{2 \pi i l \rho+2 \pi i v}\right)^{-\sum_{s=0}^{N-1} \sum_{s^{\prime}=0}^{M-1} e^{-2 \pi i l s^{\prime} / M} e^{-2 \pi i r s / N} c_{1}^{\left(0, s ; 0, s^{\prime}\right)}(-1)} \\
& \prod_{l=0}^{\infty} \prod_{r=0}^{N-1}\left(1-e^{2 \pi i r / N} e^{2 \pi i l \rho-2 \pi i v}\right)^{-\sum_{s=0}^{N-1} \sum_{s^{\prime}=0}^{M-1} e^{-2 \pi i l s^{\prime} / M} e^{-2 \pi i r s / N} c_{1}^{\left(0, s ; 0, s^{\prime}\right)}(-1)}
\end{aligned}
$$

Note that the $\left(1-e^{-2 \pi i v}\right)^{-2}$ factor has been absorbed into the $l=0$ term in the last term.

Finally let us turn to the contribution to the partition function from the motion of the D1-branes along the D5-branes. First we consider a single D1-brane wrapped $w$ times along $S^{1}$, and count the number of states $n\left(w, j, l ; r, k^{\prime}\right)$ of the system carrying left-moving momentum $l / M$ along $S^{1}, g_{M}$ eigenvalue $e^{2 \pi i k^{\prime} / M}, g_{N}$ eigenvalue $e^{2 \pi i r / N}$ and $\widetilde{S}^{1}$ momentum $j$. Since the boundary condition on various fields are twisted by $g_{M}$ under $2 \pi$ translation along $S^{1}$, the CFT on a D1-brane wrapped $w$ times along $S^{1}$ satisfies boundary condition twisted by $\left(g_{M}\right)^{w}$. Furthermore since the effective length of the D1-brane is now $2 \pi w$, a momentum $l / M$ along $S^{1}$ will appear as $l w / M$ units of momentum in the CFT living on the D1-brane. It now follows from (1.10) that $[8,42]$

$$
\begin{aligned}
n\left(w, j, l ; r, k^{\prime}\right) & =\sum_{s=0}^{N-1} \sum_{s^{\prime}=0}^{M-1} e^{-2 \pi i r s / N} e^{-2 \pi i k^{\prime} s^{\prime} / M} c_{b}^{\left(0, s ; r^{\prime}, s^{\prime}\right)}\left(4 l w / M-j^{2}\right), \\
b & =j \bmod 2, \quad r^{\prime}=w \bmod M .
\end{aligned}
$$

The requirement that we only keep the modes which are invariant under the transformation $g_{M}$ accompanied by $2 \pi$ translation along $S^{1}$ forces the constraint $k^{\prime}=l \bmod M$. It is now straightforward to evaluate the contribution to the $g_{N}$ twisted partition function from 
multiple states of this type, carrying different $(w, l, j)[46]$ :

$$
Z_{D 1 D 5}=e^{-2 \pi i \widetilde{\gamma} \sigma} \prod_{r=0}^{N-1} \prod_{b=0}^{1} \prod_{\substack{w \in \mathbb{Z}, l \in \mathbb{Z}, j \in 2 \mathbb{Z}+b \\ w>0, l \geq 0}}\left(1-e^{2 \pi i r / N} e^{2 \pi i(\sigma w / M+\rho l+v j)}\right)^{-n(w, j, l ; r, l)} .
$$

where

$$
\widetilde{\gamma}=\left\{\begin{array}{l}
1 / M \text { for } \mathcal{M}=K 3 \\
0 \text { for } \mathcal{M}=T^{4}
\end{array}\right.
$$

The $e^{-2 \pi i \widetilde{\gamma} \sigma}$ in (2.11) accounts for the fact that the actual number of D1-branes required to produce a total D1-brane charge $Q_{1}$ in the background of a D5-brane is given by $Q_{1}+1$ for $\mathcal{M}=K 3$ and $Q_{1}$ for $\mathcal{M}=T^{4}$. Multiplying (2.3), (2.9) and (2.11) we get the total partition function of the system:

$$
Z(\rho, \sigma, v)=Z_{K K} Z_{C M} Z_{D 1 D 5}=-1 / \widetilde{\Phi}(\rho, \sigma, v),
$$

where

$$
\begin{aligned}
\widetilde{\Phi}(\rho, \sigma, v) & =e^{2 \pi i(\widetilde{\alpha} \rho+\widetilde{\gamma} \sigma+\widetilde{\beta} v)} \prod_{b=0}^{1} \prod_{r=0}^{N-1} \prod_{r^{\prime}=0}^{M-1} \prod_{\substack{k \in \mathbb{Z}+\frac{r^{\prime}}{,}, l \in \mathbb{Z}, j \in 2 \mathbb{Z}+b \\
k, l \geq 0, j<0 \text { for } k=l=0}}\left[1-e^{2 \pi i r / N} e^{2 \pi i(k \sigma+l \rho+j v)}\right]^{a} \\
a & \equiv \sum_{s=0}^{N-1} \sum_{s^{\prime}=0}^{M-1} e^{-2 \pi i\left(s^{\prime} l / M+r s / N\right)} c_{b}^{\left(0, s ; r^{\prime}, s^{\prime}\right)}\left(4 k l-j^{2}\right)
\end{aligned}
$$

with $\widetilde{\alpha}, \widetilde{\beta}, \widetilde{\gamma}$ defined in (1.11). Note that the $k=0$ term in this product gives the result for $Z_{K K} Z_{C M}$.

\section{$3 \quad$ Asymptotic growth}

We now study the growth of the index for large $Q^{2}, P^{2}$ and Q.P. This can be done by standard procedure described in $[3,6,18,19]$. We deform the three dimensional contour of integration over $(\rho, \sigma, v)$ to small imaginary values of $(\rho, \sigma, v)$. During this deformation we pick up contribution from the residues at various poles, given by the zeroes of $\widetilde{\Phi}$, which give the leading contribution to the index, - the contribution from the final contour can be shown to be subleading compared to the contribution from the residues at the poles [42]. Thus we need to first determine the location of the zeroes of $\widetilde{\Phi}$. This has been done in appendix $\mathrm{C}$ where it is shown that $\widetilde{\Phi}$ has double zeroes on the subspaces:

$$
n_{2}\left(\rho \sigma-v^{2}\right)-m_{1} \rho+n_{1} \sigma+m_{2}+j v=0,
$$

for values of $\left(m_{1}, n_{1}, m_{2}, n_{2}, j\right)$ satisfying

$$
\begin{aligned}
& m_{1} n_{1}+m_{2} n_{2}+\frac{j^{2}}{4}=\frac{1}{4}, \\
& m_{1} \in M \mathbb{Z}, \quad m_{2} \in \mathbb{Z}, \quad n_{2} \in N \mathbb{Z}, \quad n_{1} \in \mathbb{Z}, \quad j \in 2 \mathbb{Z}+1 .
\end{aligned}
$$


Now the analysis of $[3,6,18,19]$ tells us that for large $Q^{2}, P^{2}, Q . P$ the contribution from the residue at the pole $(3.1)$ of $1 / \widetilde{\Phi}$ grows as

$$
\exp \left(\pi \sqrt{Q^{2} P^{2}-(Q . P)^{2}} /\left|n_{2}\right|\right) \quad \text { for }\left|n_{2}\right|>0 .
$$

On the other hand the poles at $n_{2}=0$ are responsible for wall crossing and their contribution grows much slower than (3.3)[38-41]. Thus the leading contribution comes from the pole at (3.1) for the minimum non-zero value of $\left|n_{2}\right|$. Eq. (3.2) shows that this is $N$. Thus the index grows as

$$
\exp \left(\pi \sqrt{Q^{2} P^{2}-(Q . P)^{2}} / N\right)
$$

Since for this charge the black hole entropy $S_{B H}$ is given by $\pi \sqrt{Q^{2} P^{2}-(Q \cdot P)^{2}}[10$, 11], (3.4) is in precise agreement with the macroscopic prediction (1.2).

\section{Conclusion}

It is widely believed that since string theory provides us with a consistent quantum theory of gravity, black holes in string theory do not lead to a loss of information. If so, the black hole must represent an ensemble of microstates and the black hole entropy must have an interpretation as the logarithm of the degeneracy of microstates. Furthermore quantum string theory around a black hole background must contain all possible information about the microstates. It is therefore important to learn how we can extract information about the black hole microstates by studying quantum string theory around the black hole background.

The results of [29] and this paper provide a small step in this direction. In these papers we discuss how to extract information about one specific feature of the black hole microstates, namely distribution of the $\mathbb{Z}_{N}$ charges among the microstates. Quantum string theory around the near horizon background leads to a specific algorithm for extracting this information. Our analysis shows that in the limit of large charges the results of the macroscopic analysis are in exact agreement with the microscopic results in a wide class of models where the latter is computable. While using the rules of $A d S_{2} / C F T_{1}$ correspondence we can in principle compute the ensemble average of more general operators on the black hole side, in the absence of non-renormalization results it is not clear how we might compare this with the microscopic results.

\section{Acknowledgments}

I wish to thank Nabamita Banerjee, Atish Dabholkar, Joao Gomes and Sameer Murthy for useful discussions. This work was supported in part by the JC Bose fellowship of the Department of Science and Technology, India and by the Blaise Pascal Chair, France.

Note added. I have been informed by Suresh Govandarajan that the modular forms of subgroups of $\operatorname{Sp}(2, \mathbb{Z})$ which appear here have also been constructed independently in $[47,48]$. 


\section{A Explicit computation of $c_{b}^{\left(r, s ; r^{\prime}, s^{\prime}\right)}$}

In this appendix we shall describe the strategy for explicit computation of the right hand side of (1.10)

$$
\begin{aligned}
F^{\left(r, s ; r^{\prime}, s^{\prime}\right)}(\tau, z) & \equiv \frac{1}{M N} \operatorname{Tr}_{R R ; g_{M}^{r^{\prime}} g_{N}^{r}}\left(g_{M}^{s^{\prime}} g_{N}^{s}(-1)^{J_{L}+J_{R}} e^{2 \pi i\left(\tau L_{0}-\bar{\tau} \bar{L}_{0}\right)} e^{2 \pi i J_{L} z}\right) \\
& =\sum_{b=0}^{1} \sum_{j \in 2 \mathbb{Z}+b, n \in \mathbb{Z} / M N} c_{b}^{\left(r, s ; r^{\prime}, s^{\prime}\right)}\left(4 n-j^{2}\right) e^{2 \pi i(n \tau+j z)},
\end{aligned}
$$

and hence of $c_{b}^{\left(r, s ; r^{\prime}, s^{\prime}\right)}$. First of all $F^{(0,0 ; 0,0)}$ is simply $1 / M N$ times the elliptic genus of $\mathcal{M}$ and is given by [49]

$$
\begin{aligned}
F^{(0,0 ; 0,0)}(\tau, z) & =0 \quad \text { for } \mathcal{M}=T^{4} \\
& =\frac{8}{M N}\left[\frac{\vartheta_{2}(\tau, z)^{2}}{\vartheta_{2}(\tau, 0)^{2}}+\frac{\vartheta_{3}(\tau, z)^{2}}{\vartheta_{3}(\tau, 0)^{2}}+\frac{\vartheta_{4}(\tau, z)^{2}}{\vartheta_{4}(\tau, 0)^{2}}\right] \quad \text { for } \mathcal{M}=K 3,
\end{aligned}
$$

where $\vartheta_{i}$ are the Jacobi theta functions. For non-vanishing $r$ and/or $r^{\prime}$ the contribution to the right hand side of (A.1), coming from twisted sectors localized at the fixed points of $g_{M}^{r^{\prime}} g_{N}^{r}$, can be computed by taking the size of K3 to be large so that the geometry near the fixed points is nearly flat. In this case the $\sigma$ model near the fixed point can be regarded as the orbifold of a free field theory, and the action of $g_{N}^{r} g_{M}^{r^{\prime}}$ near the fixed point may be represented by a rotation by some angle $2 \pi \theta$ in one plane and rotation by an opposite angle $-2 \pi \theta$ in the orthogonal plane. The action of $g_{M}^{s^{\prime}} g_{N}^{s}$ near such a fixed point can be of two types, - either it takes the fixed point to a different fixed point or it leaves the fixed point fixed. In the former case the contribution to the trace in (A.1) vanishes, whereas in the latter case the action of $g_{M}^{s^{\prime}} g_{N}^{s}$ near the fixed point can be represented by a rotation by $2 \pi \phi$ in one plane and a rotation by $-2 \pi \phi$ in the orthogonal plane. This gives a contribution to (A.1) of the form

$$
\begin{aligned}
& \frac{1}{M N} \prod_{n=1}^{\infty}\left\{\left(1-q^{n+\theta-1} e^{2 \pi i \phi}\right)^{-2}\left(1-q^{n-\theta} e^{-2 \pi i \phi}\right)^{-2}\left(1-q^{n+\theta-1} e^{2 \pi i \phi} e^{2 \pi i z}\right)\right. \\
&\left(1-q^{n+\theta-1} e^{2 \pi i \phi} e^{-2 \pi i z}\right)\left(1-q^{n-\theta} e^{-2 \pi i \phi} e^{2 \pi i z}\right)\left(1-q^{n-\theta} e^{-2 \pi i \phi} e^{-2 \pi i z}\right) \\
&= \frac{1}{M N} \frac{\vartheta_{1}(\tau, z+\theta \tau+\phi) \vartheta_{1}(\tau,-z+\theta \tau+\phi)}{\vartheta_{1}(\tau, \theta \tau+\phi)^{2}}
\end{aligned}
$$

where $q \equiv e^{2 \pi i \tau}$. The full contribution to $F^{\left(r, s ; r^{\prime}, s^{\prime}\right)}(\tau, z)$ is obtained by summing over the contribution from all the fixed points of $g_{N}^{r} g_{M}^{r^{\prime}}$ which are also fixed by $g_{M}^{s^{\prime}} g_{N}^{s}$. Finally $F^{\left(0, s ; 0, s^{\prime}\right)}$ can be computed using the modular transformation rules [49]

$$
F^{\left(r, s ; r^{\prime}, s^{\prime}\right)}\left(\frac{a \tau+b}{c \tau+d}, \frac{z}{c \tau+d}\right)=\exp \left[2 \pi i \frac{c z^{2}}{c \tau+d}\right] F^{\left(c s+a r, d s+b r ; c s^{\prime}+a r^{\prime}, d s^{\prime}+b r^{\prime}\right)}(\tau, z) .
$$

This gives a way to compute $F^{\left(r, s ; r^{\prime}, s^{\prime}\right)}(\tau, z)$ using purely geometric data, namely the fixed points of the different elements of $\mathbb{Z}_{M} \times \mathbb{Z}_{N}$ and the action of the elements of 
$\mathbb{Z}_{M} \times \mathbb{Z}_{N}$ near the fixed points. Note that $F^{\left(r, s ; r^{\prime}, s^{\prime}\right)}(\tau, z)$ constructed from (A.3), (A.4) is invariant under $z \rightarrow-z$. This is a consequence of the $\mathrm{SU}(2)_{L} \mathrm{R}$-symmetry of the underlying conformal field theory that allows us to change the sign of $J_{L}$.

\section{B Threshold integral representation of $\widetilde{\Phi}$}

In this appendix we shall describe a threshold integral representation of $\widetilde{\Phi}$. For this we define

$$
\begin{aligned}
h_{b}^{\left(r, s ; r^{\prime}, s^{\prime}\right)}(\tau) & =\sum_{k \in \frac{1}{M N} \mathbb{Z}-\frac{b^{2}}{4}} c_{b}^{\left(r, s ; r^{\prime}, s^{\prime}\right)}(4 k) e^{2 \pi i k \tau}, \\
\Omega & =\left(\begin{array}{ll}
\rho & v \\
v & \sigma
\end{array}\right)
\end{aligned}
$$

and

$$
\begin{aligned}
& \frac{1}{2} p_{R}^{2}=\frac{1}{4 \operatorname{det} I m \Omega}\left|-m_{1} \rho+m_{2}+n_{1} \sigma+n_{2}\left(\sigma \rho-v^{2}\right)+j v\right|^{2} \\
& \frac{1}{2} p_{L}^{2}=\frac{1}{2} p_{R}^{2}+m_{1} n_{1}+m_{2} n_{2}+\frac{1}{4} j^{2} .
\end{aligned}
$$

We now consider the 'threshold integral'

$$
\widetilde{\mathcal{I}}(\rho, \sigma, v)=\sum_{r, s=0}^{N-1} \sum_{r^{\prime}, s^{\prime}=0}^{M-1} \sum_{b=0}^{1} \widetilde{\mathcal{I}}_{r, s ; r^{\prime}, s^{\prime} ; b},
$$

where

$$
\begin{gathered}
\widetilde{\mathcal{I}}_{r, s ; r^{\prime}, s^{\prime} ; b}=\int_{\mathcal{F}} \frac{d^{2} \tau}{\tau_{2}}\left[\sum_{\substack{m_{1} \in \mathbb{Z}, m_{2} \in \mathbb{Z} / N, n_{2} \in N \mathbb{Z}-r \\
n_{1} \in \mathbb{Z}+\frac{r^{\prime}}{M}, j \in 2 \mathbb{Z}+b}} q^{p_{L}^{2} / 2} \bar{q}^{p_{R}^{2} / 2} e^{2 \pi i m_{1} s^{\prime} / M} e^{-2 \pi i m_{2} s} h_{b}^{\left(r, s ; r^{\prime}, s^{\prime}\right)}(\tau)\right. \\
\left.-\delta_{b, 0} \delta_{r, 0} \delta_{r^{\prime}, 0} c_{0}^{\left(0, s ; 0, s^{\prime}\right)}(0)\right]
\end{gathered}
$$

with

$$
q \equiv e^{2 \pi i \tau}
$$

$\mathcal{F}$ denotes the fundamental region of $\operatorname{SL}(2, \mathbb{Z})$ in the upper half plane. The subtraction terms proportional to $c_{0}^{\left(0, s ; 0, s^{\prime}\right)}(0)$ have been chosen so that the integrand vanishes faster than $1 / \tau_{2}$ in the $\tau \rightarrow i \infty$ limit, rendering the integral finite.

Following the manipulations outlined in [4] following earlier work of [50-52] one can show that

$$
\widetilde{\mathcal{I}}(\rho, \sigma, v)=-2 \ln \left[(\operatorname{det} \operatorname{Im} \Omega)^{\widetilde{k}}\right]-2 \ln \widetilde{\Phi}(\rho, \sigma, v)-2 \ln \check{\Phi}(\bar{\rho}, \bar{\sigma}, \bar{v})+\mathrm{constant}
$$


where $\widetilde{\Phi}$ has been defined in $(1.9)$ and $^{7}$

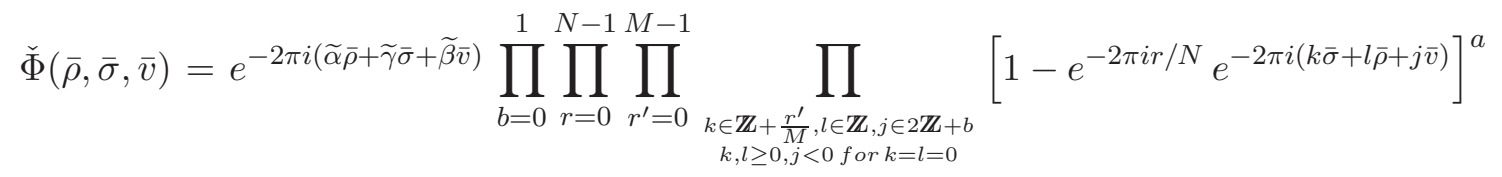

$$
\begin{aligned}
& a \equiv \sum_{s=0}^{N-1} \sum_{s^{\prime}=0}^{M-1} e^{-2 \pi i\left(s^{\prime} l / M+r s / N\right)} c_{b}^{\left(0, s ; r^{\prime}, s^{\prime}\right)}\left(4 k l-j^{2}\right) \\
& \widetilde{k}=\frac{1}{2} \sum_{s=0}^{N-1} \sum_{s^{\prime}=0}^{M-1} c_{0}^{\left(0, s ; 0, s^{\prime}\right)}(0) .
\end{aligned}
$$

Since the detailed analysis of a specific integral of this type has been carried out in [4], we shall only describe the basic steps leading to (B.7), focussing on the main differences between the general case and the special case analyzed in [4].

1. We first carry out Poisson resummation over $m_{1}$ and $m_{2}$ to express $\widetilde{\mathcal{I}}_{r, s ; r^{\prime}, s^{\prime} ; b}$ as

$$
\int_{\mathcal{F}} \frac{d^{2} \tau}{\tau_{2}^{2}} \frac{Y}{\rho_{2}}\left[N \sum_{\substack{k_{1} \in \mathbb{Z}+\frac{s^{\prime}}{M}, k_{2} \in N \mathbb{Z}-s \\ n_{1} \in \mathbb{Z}+\frac{r^{\prime}}{M}, n_{2} \in N \mathbb{Z}-r, j \in 2 \mathbb{Z}+b}} h_{b}^{\left(r, s ; r^{\prime}, s^{\prime}\right)}(\tau) e^{\mathcal{G}(\vec{n}, \vec{k}, j)}-\delta_{b, 0} \delta_{r, 0} \delta_{r^{\prime}, 0} c_{0}^{\left(0, s ; 0, s^{\prime}\right)}(0)\right]
$$

where

$$
\begin{aligned}
\mathcal{G}(\vec{n}, \vec{k}, j)= & -\frac{\pi Y}{\rho_{2}^{2} \tau_{2}}|\mathcal{A}|^{2}-2 \pi i \sigma \operatorname{det} A+\frac{\pi j}{\rho_{2}}(v \tilde{\mathcal{A}}-\bar{v} \mathcal{A})-\frac{\pi n_{2}}{\rho_{2}}\left(v^{2} \tilde{\mathcal{A}}-\bar{v}^{2} \mathcal{A}\right) \\
& +\frac{2 \pi i v_{2}^{2}}{\rho_{2}^{2}}\left(n_{1}+n_{2} \bar{\rho}\right) \mathcal{A}+2 \pi i \tau \frac{j^{2}}{4} \\
Y \equiv & \rho_{2} \sigma_{2}-v_{2}^{2}, A \equiv\left(\begin{array}{ll}
n_{1} & k_{1} \\
n_{2} & k_{2}
\end{array}\right), \mathcal{A} \equiv(1, \rho) A\left(\begin{array}{l}
\tau \\
1
\end{array}\right), \tilde{\mathcal{A}} \equiv(1, \bar{\rho}) A\left(\begin{array}{l}
\tau \\
1
\end{array}\right) .
\end{aligned}
$$

2. Using (B.4), (B.10) and performing the sum over $j$ using (A.1), (B.1) we get

$$
\widetilde{\mathcal{I}}=\int_{\mathcal{F}} \frac{d^{2} \tau}{\tau_{2}^{2}}\left[N \sum_{k_{1}, n_{1} \in \frac{\mathbb{Z}}{M}, k_{2}, n_{2} \in \mathbb{Z}} \mathcal{J}(A, \tau)-\sum_{s=0}^{N-1} \sum_{s^{\prime}=0}^{M-1} c_{0}^{\left(0, s ; 0, s^{\prime}\right)}(0)\right],
$$

where

$$
\begin{aligned}
\mathcal{J}(A, \tau)= & \frac{Y}{\rho_{2}} \exp \left(-\frac{\pi Y}{\rho_{2}^{2} \tau_{2}}|\mathcal{A}|^{2}-2 \pi i \sigma \operatorname{det} A\right. \\
& \left.-\frac{\pi n_{2}}{\rho_{2}}\left(v^{2} \tilde{\mathcal{A}}-\bar{v}^{2} \mathcal{A}\right)+\frac{2 \pi i v_{2}^{2}}{\rho_{2}^{2}}\left(n_{1}+n_{2} \bar{\rho}\right) \mathcal{A}\right) F^{\left(r, s ; r^{\prime}, s^{\prime}\right)}\left(\tau,-i \frac{v \tilde{\mathcal{A}}-\bar{v} \mathcal{A}}{2 \rho_{2}}\right)
\end{aligned}
$$

$r=-n_{2} \bmod N, s=-k_{2} \bmod N, r^{\prime}=M n_{1} \bmod M, s^{\prime}=M k_{1} \bmod M$.

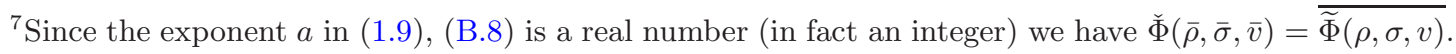


3. Using (A.4) one can show that

$$
\mathcal{J}\left(A\left(\begin{array}{ll}
a & b \\
c & d
\end{array}\right), \tau\right)=\mathcal{J}\left(A, \frac{a \tau+b}{c \tau+d}\right), \quad\left(\begin{array}{ll}
a & b \\
c & d
\end{array}\right) \in \operatorname{SL}(2, \mathbb{Z}) .
$$

Under the map $\tau \rightarrow(a \tau+b) /(c \tau+d)$ the fundamental region $\mathcal{F}$ of $\operatorname{SL}(2, \mathbb{Z})$ gets mapped to its image. With the help of (B.15) we can restrict the sum over the matrix $A$ to the following ranges [50]:

(a) Non-degenerate orbit:

$$
A=\left(\begin{array}{cc}
k & m \\
0 & p
\end{array}\right), \quad k, m \in \frac{\mathbb{Z}}{M}, \quad p \in \mathbb{Z}, \quad p \neq 0, \quad 0 \leq m<k,
$$

with the integration over $\tau$ ranging over two copies of the upper half plane.

(b) Degenerate orbit:

$$
A=\left(\begin{array}{cc}
0 & m \\
0 & p
\end{array}\right), \quad m \in \frac{\mathbb{Z}}{M}, \quad p \in \mathbb{Z}, \quad(m, p) \neq(0,0),
$$

with the integration over $\tau$ ranging over the strip $-\frac{1}{2} \leq \tau_{1} \leq \frac{1}{2}, \tau_{2}>0$.

(c) Zero orbit:

$$
A=\left(\begin{array}{ll}
0 & 0 \\
0 & 0
\end{array}\right),
$$

with the integration over $\tau$ ranging over the fundamental domain $\mathcal{F}$.

We shall briefly discuss the computation of the contribution from the non-degenerate orbits. The analysis of the contribution from the other orbits follows [4].

4. The steps needed for evaluating the contribution from the non-degenerate orbits are as follows:

(a) We first expand $F^{\left(r, s ; r^{\prime}, s^{\prime}\right)}(\tau, z)$ using (A.1) and change integration variable from $\tau_{1}$ to $\tau_{1}^{\prime}$ :

$$
\tau_{1}^{\prime}=\tau_{1}+\frac{m}{k}+\frac{p}{k} \rho_{1} .
$$

Since for non-degenerate orbit the $\tau_{1}$ integration ranges from $-\infty$ to $\infty, \tau_{1}^{\prime}$ integral will also range from $-\infty$ to $\infty$.

(b) After this change of variables the $m$ dependence of the integral becomes simple. The explicit dependence on $m$ takes the form $e^{-2 \pi i m n / k}, n$ and $j$ being the integers appearing in the expansion (A.1). There is also an implicit dependence on $m$ through $c_{b}^{\left(r, s ; r^{\prime}, s^{\prime}\right)}\left(4 n-j^{2}\right)$ since $s^{\prime}=M m \bmod M$ due to (B.14). The sum over $m$ can be performed by expanding the summation range of $m$ to $0 \leq m<M k$ in steps of $1 / M$, at the cost of having to divide the sum by a factor of $M$. We then sum over all values of $m$ of the form $\frac{s^{\prime}}{M}+$ integer for fixed 
$s^{\prime}$ and finally sum over integral $s^{\prime}$ in the range $0 \leq s^{\prime} \leq(M-1)$. For fixed $s^{\prime}$ the sum takes the form

$$
\sum_{m \in \mathbb{Z}+\frac{s^{\prime}}{M}, 0 \leq m<M k-1} e^{-2 \pi i m n / k}=M k e^{-2 \pi i n s^{\prime} /(M k)} \sum_{l \in \mathbb{Z}} \delta_{n, k l}=M k \sum_{l \in \mathbb{Z}} e^{-2 \pi i l s^{\prime} / M} \delta_{n, k l} .
$$

The sum over $n$ can now be performed using the Kronecker delta.

(c) Next we carry out the $\tau_{1}^{\prime}$ integral which is Gaussian and the $\tau_{2}$ integral using the identity

$$
\int_{0}^{\infty} \frac{d u}{u^{3 / 2}} e^{-a u-b u^{-1}}=\sqrt{\frac{\pi}{b}} e^{-2 \sqrt{a b}} .
$$

At this stage the $p$ dependent part of the summand takes the form

$$
\frac{1}{|p|} \exp \left\{-2 \pi i \sigma k p-2 \pi k|p| \sigma_{2}-2 \pi k p \sigma_{2}-2 \pi i l p \rho_{1}-2 \pi l|p| \rho_{2}-2 \pi i j p v_{1}-2 \pi j|p| v_{2}\right\} \text {. }
$$

(d) Finally we perform the sum over $p$ by breaking it into contribution from $p>0$ and $p<0$ terms, making a change of variables $p \rightarrow-p$ in the $p<0$ terms, and using the identity

$$
\begin{aligned}
\sum_{p \in N \mathbb{Z}+s, p>0} \frac{1}{p} e^{2 \pi i \alpha p} & =\frac{1}{N} \sum_{r=0}^{N-1} \sum_{p \in \mathbb{Z}, p>0} e^{2 \pi i r(p-s) / N} \frac{1}{p} e^{2 \pi i \alpha p} \\
& =-\frac{1}{N} \sum_{r=0}^{N-1} e^{-2 \pi i r s / N} \ln \left(1-e^{2 \pi i r / N} e^{2 \pi i \alpha}\right) .
\end{aligned}
$$

5. At the end of this manipulation we get the contribution from the non-degenerate orbits to be

$$
\begin{aligned}
-2 & \sum_{r, s=0}^{N-1} \sum_{r^{\prime}, s^{\prime}=0}^{M-1} \sum_{b=0}^{1} \sum_{k \in \mathbb{Z}+\frac{r^{\prime}}{M}, l \in \mathbb{Z}, j \in 2 \mathbb{Z}+b, k>0, l \geq 0} e^{-2 \pi i r s / N} e^{-2 \pi i l s^{\prime} / M} c_{b}^{\left(0, s ; r^{\prime}, s^{\prime}\right)}\left(4 k l-j^{2}\right) \\
& \left\{\ln \left[1-e^{2 \pi i r / N} e^{2 \pi i(k \sigma+l \rho+j v)}\right]+\ln \left[1-e^{-2 \pi i r / N} e^{-2 \pi i(k \bar{\sigma}+l \bar{\rho}+j \bar{v})}\right]\right\}+\text { constant } .
\end{aligned}
$$

One can recognize (B.24) as the contribution from $-2 \ln \widetilde{\Phi}(\rho, \sigma, v)-2 \ln \check{\Phi}(\bar{\rho}, \bar{\sigma}, \bar{v})$ except for the $k=0$ terms and the overall multiplicative factors in the product representation (1.9) of $\widetilde{\Phi}$ and (B.8) of $\check{\Phi}$. By carefully analyzing the contribution from the degenerate and the zero orbits one recovers the complete set of terms on the left hand side of (B.7).

\section{Zeroes of $\widetilde{\Phi}$}

In this appendix we shall determine the locations of the zeroes of the function $\widetilde{\Phi}$. From (B.7) it follows that at the locations of the zeroes and poles of $\widetilde{\Phi}$ we have logarithmic divergences in $\widetilde{\mathcal{I}}$. Thus we can determine the locations of the zeroes and poles of $\widetilde{\Phi}$ by determining 
the singularities of $\widetilde{\mathcal{I}}$. Since the integrand in $\widetilde{\mathcal{I}}$ is finite for finite $\tau$, and in its original form given in (B.4), (B.5) the integration over $\tau$ runs over the fundamental domain of $\mathrm{SL}(2, \mathbb{Z})$, the only possible source of divergence is from the region of large $\tau_{2}$. This requires that the powers of $q\left(\equiv e^{2 \pi i \tau}\right)$ and $\bar{q}$ be equal so that the $\tau_{1}$ integral for large $\tau_{2}$ does not vanish, and non-positive so that the $\tau_{2}$ integral diverges. Since the only dependence on $\bar{q}$ is through the $\bar{q}^{p_{R}^{2} / 2}$ term, and since $p_{R}^{2}$ is positive semi-definite, this requires $p_{R}^{2}$ to vanish. Furthermore $p_{L}^{2}$ is also positive semidefinite (although it is not directly apparent from its definition), and hence the only possible way to produce a divergence is to get a non-positive power of $q$ from the expansion of $h_{b}^{\left(r, s ; r^{\prime}, s^{\prime}\right)}(\tau) .^{8}$ Such terms are quite restricted, and one finds that the possible divergences in $\widetilde{\mathcal{I}}$ arise at $[6,42]$

$$
n_{2}\left(\rho \sigma-v^{2}\right)-m_{1} \rho+n_{1} \sigma+m_{2}+j v=0,
$$

for values of $\left(m_{1}, n_{1}, m_{2}, n_{2}, j\right)$ satisfying

$$
\begin{aligned}
& m_{1} n_{1}+m_{2} n_{2}+\frac{j^{2}}{4}=\frac{1}{4}-\beta, \quad 0 \leq \beta<\frac{1}{4}, \\
& m_{1} \in \mathbb{Z}, \quad m_{2} \in \mathbb{Z} / N, \quad n_{2} \in \mathbb{Z}, \quad n_{1} \in \mathbb{Z} / M, \quad j \in 2 \mathbb{Z}+1 .
\end{aligned}
$$

At this point $p_{R}^{2}$ vanishes and $p_{L}^{2} / 2=(1 / 4)-\beta$ so that the $q^{p_{L}^{2} / 2} \bar{q}^{p_{R}^{2} / 2}$ factor becomes purely a function of $\tau$ and not of $\bar{\tau}$. This has to be cancelled against a similar $\tau$ dependent factor in $h_{b}$ so that the $\tau_{1}$ integral is finite and we get a logarithmically divergent $\tau_{2}$ integral. The coefficient of the divergent term can be easily determined from (B.5), (B.1) and (B.7) and we get, near this point, ${ }^{9}$

$$
\begin{aligned}
\widetilde{\Phi}(\rho, \sigma, v) & \sim\left(n_{2}\left(\rho \sigma-v^{2}\right)-m_{1} \rho+n_{1} \sigma+m_{2}+j v\right)^{\sum_{s^{\prime}=0}^{M-1} \sum_{s=0}^{N-1} e^{2 \pi i m_{1} s^{\prime} / M} e^{-2 \pi i m_{2} s} c_{1}^{\left(r, s ; r^{\prime}, s^{\prime}\right)}(-1+4 \beta),} \\
r & =-n_{2} \bmod N, \quad r^{\prime}=M n_{1} \bmod M .
\end{aligned}
$$

Let us first focus on the $r=r^{\prime}=0$ terms, i.e. configurations with $n_{2} \in N \mathbb{Z}, n_{1} \in \mathbb{Z}$. In this case it follows from (C.2) that $\beta$ must vanish and so the right hand side of (C.3) involves $c_{1}^{\left(0, s ; 0, s^{\prime}\right)}(-1)$. Using $(2.8)$ we now get, for $\mathcal{M}=K 3$,

$$
\sum_{s^{\prime}=0}^{M-1} \sum_{s=0}^{N-1} e^{2 \pi i m_{1} s^{\prime} / M} e^{-2 \pi i m_{2} s} c_{1}^{\left(0, s ; 0, s^{\prime}\right)}(-1)=\left\{\begin{array}{l}
2 \text { for } m_{1} \in M \mathbb{Z}, m_{2} \in \mathbb{Z} \\
0 \text { otherwise }
\end{array}\right.
$$

and for $\mathcal{M}=T^{4}$,

$$
\sum_{s^{\prime}=0}^{M-1} \sum_{s=0}^{N-1} e^{2 \pi i m_{1} s^{\prime} / M} e^{-2 \pi i m_{2} s} c_{1}^{\left(0, s ; 0, s^{\prime}\right)}(-1)=\left\{\begin{array}{l}
2 \text { for } m_{1} \in M \mathbb{Z}, m_{2} \in \mathbb{Z} \\
-1 \text { for } m_{1} \in M \mathbb{Z} \pm 1, m_{2} \in \mathbb{Z} \mp \frac{1}{N} \\
0 \text { otherwise }
\end{array}\right.
$$

\footnotetext{
${ }^{8}$ There is a further restriction coming from the fact that for $p_{L}^{2}=p_{R}^{2}=0$ all the $m_{i}$ 's, $n_{i}$ 's and $j$ must vanish. The divergence in the $\tau_{2}$ integral from such a term is removed by the subtraction term in (B.5). Thus a divergent $\tau_{2}$ integral requires $p_{L}^{2}$ to be strictly positive. This explains the strict inequality $\beta<\frac{1}{4}$ in (C.2).

${ }^{9}$ We need to account for the fact that the terms in the expression for $\widetilde{\mathcal{I}}$ with $\left(m_{i}, n_{i}, j\right)$ and $\left(-m_{i},-n_{i},-j\right)$ give identical results.
} 
In either case the exponent is positive, producing zeroes of $\widetilde{\Phi}$, only for $m_{1} \in M \mathbb{Z}$ and $m_{2} \in \mathbb{Z}$. Thus the net result is that the only zeroes of $\widetilde{\Phi}$ for $r=r^{\prime}=0$ are of the form:

$$
\begin{aligned}
\widetilde{\Phi}(\rho, \sigma, v) \sim & \left(n_{2}\left(\rho \sigma-v^{2}\right)-m_{1} \rho+n_{1} \sigma+m_{2}+j v\right)^{2}, \\
& m_{1} \in M \mathbb{Z}, \quad m_{2} \in \mathbb{Z}, \quad n_{1} \in \mathbb{Z}, \quad n_{2} \in N \mathbb{Z} .
\end{aligned}
$$

Let us now analyze the contribution to the exponent in (C.3) when $r$ and/or $r^{\prime}$ is non-zero. From the definition of the coefficients $c_{b}^{\left(r, s ; r^{\prime}, s^{\prime}\right)}$ given in (1.10) it follows that the exponent in (C.3) can be interpreted as the number of states weighted by $(-1)^{J_{L}+J_{R}}$ in the sector twisted by $g_{N}^{r} g_{M}^{r^{\prime}}$, and carrying $g_{M}$ eigenvalue $e^{-2 \pi i m_{1} / M}, g_{N}$ eigenvalue $e^{2 \pi i m_{2}}, J_{L}= \pm 1$, $L_{0}=\beta$ and $\bar{L}_{0}=0$. If $g_{M}^{r^{\prime}} g_{N}^{r}$ does not have a fixed point in $\mathcal{M}$ then this number is zero since by taking the size of $\mathcal{M}$ to be sufficiently large we can ensure that there will be no twisted sector state with $\bar{L}_{0}=0$. If $g_{M}^{r^{\prime}} g_{N}^{r}$ has fixed points in $\mathcal{M}$, then we can compute this number by taking the size of $\mathcal{M}$ to be large so that near the fixed points we can regard the space as almost flat, with $g_{N}^{r} g_{M}^{r^{\prime}}$ acting as rotation by some angle $\theta$ in one plane and by $-\theta$ in an orthogonal plane. Under such rotations all the bosons and fermions in the sigma model with target space $\mathcal{M}$ are twisted and hence there are no zero modes. Thus we have a unique ground state with $\left(J_{L}=J_{R}=0\right)$. Even after we apply left-moving oscillators

to create states with $L_{0}=\beta, J_{L}= \pm 1$, the states will continue to have $J_{R}=0$. Thus the weight factor $(-1)^{J_{L}+J_{R}}$ is always -1 for $J_{L}= \pm 1$ and hence the exponent of (C.3) is always negative. This shows that (C.3) never gives a zero of $\widetilde{\Phi}$ for $r$ and/or $r^{\prime}$ non-zero, and the only zeroes of $\widetilde{\Phi}$ are of the form given in (C.6).

Open Access. This article is distributed under the terms of the Creative Commons Attribution Noncommercial License which permits any noncommercial use, distribution, and reproduction in any medium, provided the original author(s) and source are credited.

\section{References}

[1] S. Chaudhuri, G. Hockney and J.D. Lykken, Maximally supersymmetric string theories in $D<10$, Phys. Rev. Lett. 75 (1995) 2264 [hep-th/9505054] [SPIRES].

[2] S. Chaudhuri and J. Polchinski, Moduli space of CHL strings, Phys. Rev. D 52 (1995) 7168 [hep-th/9506048] [SPIRES].

[3] D.P. Jatkar and A. Sen, Dyon spectrum in CHL models, JHEP 04 (2006) 018 [hep-th/0510147] [SPIRES].

[4] J.R. David, D.P. Jatkar and A. Sen, Product representation of dyon partition function in CHL models, JHEP 06 (2006) 064 [hep-th/0602254] [SPIRES];

[5] A. Dabholkar and S. Nampuri, Spectrum of dyons and black holes in CHL orbifolds using borcherds lift, JHEP 11 (2007) 077 [hep-th/0603066] [SPIRES].

[6] J.R. David and A. Sen, CHL dyons and statistical entropy function from D1 - D5 system, JHEP 11 (2006) 072 [hep-th/0605210] [SPIRES].

[7] J.R. David, D.P. Jatkar and A. Sen, Dyon spectrum in $N=4$ supersymmetric type-II string theories, JHEP 11 (2006) 073 [hep-th/0607155] [SPIRES]. 
[8] J.R. David, D.P. Jatkar and A. Sen, Dyon spectrum in generic $N=4$ supersymmetric $Z(N)$ orbifolds, JHEP 01 (2007) 016 [hep-th/0609109] [SPIRES].

[9] A. Dabholkar and D. Gaiotto, Spectrum of CHL dyons from genus-two partition function, JHEP 12 (2007) 087 [hep-th/0612011] [SPIRES].

[10] M. Cvetič and D. Youm, Dyonic BPS saturated black holes of heterotic string on a six torus, Phys. Rev. D 53 (1996) 584 [hep-th/9507090] [SPIRES].

[11] M. Cvetič and A.A. Tseytlin, Solitonic strings and BPS saturated dyonic black holes, Phys. Rev. D 53 (1996) 5619 [Erratum ibid. D 55 (1997) 3907] [hep-th/9512031] [SPIRES].

[12] A. Strominger and C. Vafa, Microscopic origin of the Bekenstein-Hawking entropy, Phys. Lett. B 379 (1996) 99 [hep-th/9601029] [SPIRES].

[13] J.C. Breckenridge, R.C. Myers, A.W. Peet and C. Vafa, D-branes and spinning black holes, Phys. Lett. B 391 (1997) 93 [hep-th/9602065] [SPIRES].

[14] B. de Wit, $N=2$ electric-magnetic duality in a chiral background, Nucl. Phys. Proc. Suppl. 49 (1996) 191 [hep-th/9602060] [SPIRES].

[15] B. de Wit, N=2 symplectic reparametrizations in a chiral background, Fortsch. Phys. 44 (1996) 529 [hep-th/9603191] [SPIRES].

[16] G. Lopes Cardoso, B. de Wit and T. Mohaupt, Corrections to macroscopic supersymmetric black-hole entropy, Phys. Lett. B 451 (1999) 309 [hep-th/9812082] [SPIRES].

[17] T. Mohaupt, Black hole entropy, special geometry and strings, Fortsch. Phys. 49 (2001) 3 [hep-th/0007195] [SPIRES].

[18] R. Dijkgraaf, E.P. Verlinde and H.L. Verlinde, Counting dyons in $N=4$ string theory, Nucl. Phys. B 484 (1997) 543 [hep-th/9607026] [SPIRES].

[19] G. Lopes Cardoso, B. de Wit, J. Kappeli and T. Mohaupt, Asymptotic degeneracy of dyonic $N=4$ string states and black hole entropy, JHEP 12 (2004) 075 [hep-th/0412287] [SPIRES].

[20] D. Shih, A. Strominger and X. Yin, Recounting dyons in $N=4$ string theory, JHEP 10 (2006) 087 [hep-th/0505094] [SPIRES].

[21] D. Gaiotto, Re-recounting dyons in $N=4$ string theory, hep-th/0506249 [SPIRES].

[22] D. Shih and X. Yin, Exact black hole degeneracies and the topological string, JHEP 04 (2006) 034 [hep-th/0508174] [SPIRES].

[23] N. Banerjee, D.P. Jatkar and A. Sen, Adding charges to $N=4$ dyons, JHEP 07 (2007) 024 [arXiv: 0705.1433] [SPIRES].

[24] S. Banerjee, A. Sen and Y.K. Srivastava, Generalities of quarter BPS dyon partition function and dyons of torsion two, JHEP 05 (2008) 101 [arXiv:0802.0544] [SPIRES].

[25] S. Banerjee, A. Sen and Y.K. Srivastava, Partition functions of torsion i1 dyons in heterotic string theory on $T^{6}$, JHEP 05 (2008) 098 [arXiv: 0802.1556] [SPIRES].

[26] A. Dabholkar, J. Gomes and S. Murthy, Counting all dyons in $N=4$ string theory, arXiv:0803.2692 [SPIRES].

[27] A. Sen, Quantum entropy function from $A d S_{2} / C F T_{1}$ correspondence, Int. J. Mod. Phys. A 24 (2009) 4225 [arXiv:0809.3304] [SPIRES].

[28] A. Sen, Arithmetic of quantum entropy function, JHEP 08 (2009) 068 [arXiv:0903.1477] [SPIRES]. 
[29] A. Sen, A twist in the dyon partition function, JHEP 05 (2010) 028 [arXiv:0911.1563] [SPIRES].

[30] M.G. Alford, J. March-Russell and F. Wilczek, Discrete quantum hair on clack holes and the nonabelian Aharonov-Bohm effect, Nucl. Phys. B 337 (1990) 695 [SPIRES].

[31] S.R. Coleman, J. Preskill and F. Wilczek, Quantum hair on black holes, Nucl. Phys. B 378 (1992) 175 [hep-th/9201059] [SPIRES].

[32] A. Gregori et al., $R^{2}$ corrections and non-perturbative dualities of $N=4$ string ground states, Nucl. Phys. B 510 (1998) 423 [hep-th/9708062] [SPIRES].

[33] E. Kiritsis, Introduction to non-perturbative string theory, hep-th/9708130 [SPIRES].

[34] N. Banerjee, D.P. Jatkar and A. Sen, Asymptotic expansion of the $N=4$ dyon degeneracy, JHEP 05 (2009) 121 [arXiv:0810.3472] [SPIRES].

[35] S. Murthy and B. Pioline, A farey tale for $N=4$ dyons, JHEP 09 (2009) 022 [arXiv:0904.4253] [SPIRES].

[36] S. Chaudhuri and D.A. Lowe, Type IIA heterotic duals with maximal supersymmetry, Nucl. Phys. B 459 (1996) 113 [hep-th/9508144] [SPIRES].

[37] P.S. Aspinwall, Some relationships between dualities in string theory, Nucl. Phys. Proc. Suppl. 46 (1996) 30 [hep-th/9508154] [SPIRES].

[38] A. Sen, Walls of marginal stability and dyon spectrum in $N=4$ supersymmetric string theories, JHEP 05 (2007) 039 [hep-th/0702141] [SPIRES].

[39] A. Dabholkar, D. Gaiotto and S. Nampuri, Comments on the spectrum of CHL dyons, JHEP 01 (2008) 023 [hep-th/0702150] [SPIRES].

[40] A. Sen, Two centered black holes and $N=4$ dyon spectrum, JHEP 09 (2007) 045 [arXiv:0705.3874] [SPIRES].

[41] M.C.N. Cheng and E. Verlinde, Dying dyons don't count, JHEP 09 (2007) 070 [arXiv:0706.2363] [SPIRES].

[42] A. Sen, Black hole entropy function, attractors and precision counting of microstates, Gen. Rel. Grav. 40 (2008) 2249 [arXiv:0708.1270] [SPIRES].

[43] C.N. Pope, Axial vector anomalies and the index theorem in charged Schwarzschild and Taub-nut spaces, Nucl. Phys. B 141 (1978) 432 [SPIRES].

[44] J.P. Gauntlett, N. Kim, J. Park and P. Yi, Monopole dynamics and BPS dyons in $N=2$ super-Yang-Mills theories, Phys. Rev. D 61 (2000) 125012 [hep-th/9912082] [SPIRES].

[45] D. Gaiotto, A. Strominger and X. Yin, New connections between $4 D$ and 5D black holes, JHEP 02 (2006) 024 [hep-th/0503217] [SPIRES].

[46] R. Dijkgraaf, G.W. Moore, E.P. Verlinde and H.L. Verlinde, Elliptic genera of symmetric products and second quantized strings, Commun. Math. Phys. 185 (1997) 197 [hep-th/9608096] [SPIRES].

[47] S. Govindarajan, New Lie algebras from dyonic black holes, talk at NSM 2010, IIT Bombay.

[48] S. Govindarajan, BKM Lie superalgebras from twisted CHL dyons, to appear.

[49] T. Kawai, Y. Yamada and S.-K. Yang, Elliptic genera and $N=2$ superconformal field theory, Nucl. Phys. B 414 (1994) 191 [hep-th/9306096] [SPIRES]. 
[50] L.J. Dixon, V. Kaplunovsky and J. Louis, Moduli dependence of string loop corrections to gauge coupling constants, Nucl. Phys. B 355 (1991) 649 [SPIRES].

[51] T. Kawai, $N=2$ heterotic string threshold correction, $K 3$ surface and generalized Kac-Moody superalgebra, Phys. Lett. B 372 (1996) 59 [hep-th/9512046] [SPIRES].

[52] C.D.D. Neumann, The elliptic genus of Calabi-Yau 3-folds and 4-folds, product formulae and generalized Kac-Moody algebras, J. Geom. Phys. 29 (1999) 5 [hep-th/9607029] [SPIRES]. 